\title{
ABOUT SOME CATEGORIES OF SEGAL TOPOLOGICAL ALGEBRAS
}

\author{
MART ABEL
}

\author{
Date of Receiving : $\quad 01.05 .2018$ \\ Date of Revision : $\quad 21.02 .2019$ \\ Date of Acceptance : 21.02 .2019
}

\begin{abstract}
We will construct two categories of Segal topological algebras and prove some of their categorical properties. We will show that several properties known for categories (of sets, for example) have analogues in the category $\mathscr{S}(B)$ of Segal topological algebras.
\end{abstract}

\section{Introduction}

The study of Segal topological algebras in such generality started in [3]. We will start with recalling the necessary definitions from [3].

By a topological algebra we will mean a topological linear space over the field $\mathbb{K}$ (here $\mathbb{K}$ could be either the field $\mathbb{R}$ of real numbers or the field $\mathbb{C}$ of complex numbers), in which is defined a separately continuous (associative, but not necessarily commutative) multiplication.

A topological algebra $\left(A, \tau_{A}\right)$ is a left (right or two-sided) Segal topological algebra in a topological algebra $\left(B, \tau_{B}\right)$ via an algebra homomorphism $f: A \rightarrow B$, if

1) $\operatorname{cl}_{B}(f(A))=B$

2) $\tau_{A} \supseteq\left\{f^{-1}(U): U \in \tau_{B}\right\}$;

3) $f(A)$ is a left (respectively, right or two-sided) ideal of $B$.

In short, we will denote a Segal topological algebra by a triple $(A, f, B)$.

As usual, we denote for a category $\mathscr{C}$ the set of objects of $\mathscr{C}$ by $\mathrm{Ob}(\mathscr{C})$ and for every $A, B \in \mathrm{Ob}(\mathscr{C})$ the set of morphisms from $A$ to $B$ by $\operatorname{Mor}(A, B)$.

We will offer two constructions of the categories of Segal topological algebras, denoted by Seg and $\mathscr{S}(B)$, where the objects of the second category are also objects in the first

2010 Mathematics Subject Classification. 46H05, 18Axx.

Key words and phrases. Segal topological algebras, category, equalizers, coequalizers, pullbacks.

The research was supported by the institutional research funding IUT20-57 of the Estonian Ministry of Education and Research.

Communicated by: P.P. Ntumba 